\title{
Examination of the Hipparcos Proper Motion System from Lunar Occultation Analysis
}

\author{
Mitsuru Sôma \\ National Astronomical Observatory, Mitaka, Tokyo 181-8588, Japan \\ somamtecc.nao.ac.jp
}

\begin{abstract}
Lunar occultations were analyzed to detect errors in the proper motion system of the Hipparcos frame. The analysis suggests much larger errors in the rotation of the Hipparcos reference frame than those explained by the Hipparcos team, which were determined from the direct comparison between the Hipparcos and FK5 catalogs.
\end{abstract}

\section{Introduction}

The Fundamental Catalog FK5 (Fricke et al., 1988) had been used for positions of objects on the sky, but it is well-known that the IAU 1976 precession constant used in the FK5 has an error $\Delta p$ of about -3.0 mas/year, which had been independently obtained from VLBI (McCarthy \& Luzum, 1991), lunar laser ranging (Williams et al., 1991), and proper motion analyses (Miyamoto \& Sôma, 1993). Therefore one can expect that the FK5 system should have the following rotation with respect to the ICRS (International Celestial Reference System):

$$
\begin{array}{ll}
\omega_{x} & \sim 0 \\
\omega_{y}=-\Delta p \sin \epsilon & \sim+1.2 \mathrm{mas} / \text { year } \\
\omega_{z}=\Delta p \cos \epsilon-\Delta E & \sim-1.6 \mathrm{mas} / \text { year }
\end{array}
$$

where we use the value of the equinox motion $\Delta E$ of about -1.2 mas/year by Miyamoto \& Sôma (1993).

The Hipparcos Catalogue (ESA, 1997) was released in 1997. The Hipparcos reference frame is said to be linked to the ICRS with the accuracy of 0.6 mas (milliarcseconds) in orientation and 0.25 mas/year in spin, but the direct comparison of the proper motions between Hipparcos and FK5 made by Feissel and Mignard (1998) gives the following rotation of the FK5 system with respect to the Hipparcos system:

$$
\begin{aligned}
& \omega_{x}=-0.30 \mathrm{mas} / \text { year } \\
& \omega_{y}=+0.60 \mathrm{mas} / \text { year } \\
& \omega_{z}=+0.70 \mathrm{mas} / \text { year }
\end{aligned}
$$

which is inconsistent with equation (1).

Therefore it is important to evaluate the accuracy of the Hipparcos proper motion system by other methods. Now that the lunar positions in the latest 
JPL lunar and planetary ephemerides DE405 (Standish, 1998) have mas-level accuracy with respect to the ICRS because of the very high accuracy of the lunar laser ranging measurements, the problem can be resolved by measuring the lunar positions with respect to the Hipparcos stars. The author analyzed lunar occultation observations made from 1955 using the DE405 ephemerides and the Hipparcos and ACT catalogs to detect the possible errors in the Hipparcos proper motion system. This paper discusses the results of the analysis.

\section{Observations of Lunar Occultations}

Lunar occultation observations were collected until 1980 by RGO (Royal Greenwich Observatory) and after that by ILOC (International Lunar Occultation Center) in Tokyo. The ILOC released the observations made by the end of 1997. Since it is important to convert the observed Universal Time to a uniform time scale (TAI in this case) in the analysis, only the observations made after 1955.5 are analyzed in this paper. In addition, visual observations suffer from personal equations (observers' reaction times) and can give secular effects in the analysis. Therefore we only deal with observations made photoelectrically or by video equipment.

The total number of such observations is 15,430 . The number of such observations in each year is given in Table 1 .

Table 1. Number of Photoelectric and Video Observations.

\begin{tabular}{|rr||rr||rr||cc||cc|}
\hline Year & No. & Year & No. & Year & No. & Year & No. & Year & No. \\
\hline & & 1961 & 60 & 1971 & 369 & 1981 & 528 & 1991 & 549 \\
& & 1962 & 15 & 1972 & 517 & 1982 & 571 & 1992 & 674 \\
& & 1963 & 11 & 1973 & 516 & 1983 & 779 & 1993 & 619 \\
& & 1964 & 33 & 1974 & 395 & 1984 & 611 & 1994 & 543 \\
1955 & 10 & 1965 & 33 & 1975 & 515 & 1985 & 557 & 1995 & 904 \\
1956 & 57 & 1966 & 26 & 1976 & 502 & 1986 & 471 & 1996 & 616 \\
1957 & 34 & 1967 & 63 & 1977 & 695 & 1987 & 478 & 1997 & 633 \\
1958 & 21 & 1968 & 97 & 1978 & 559 & 1988 & 395 & & \\
1959 & 21 & 1969 & 200 & 1979 & 393 & 1989 & 467 & & \\
1960 & 59 & 1970 & 260 & 1980 & 143 & 1990 & 431 & & \\
\hline
\end{tabular}

\section{Analysis}

The analysis were made using the following parameters based on the analysis by Sôma (1985):

Corrections to the Moon's positions: $\Delta \lambda=+0$ " 50 and $\Delta \beta=-0$ !'24

Radius of the Moon: $R_{0}=1738.107 \mathrm{~km}$

Correction for the ellipticity of Watts' datum: $\Delta \rho=+0$ "' $128 \sin 2$ (WA)

Relation of Watts Angle (WA) with Axis Angle (AA): WA $=\mathrm{AA}+0^{\circ} .24$

The values $\Delta \lambda$ and $\Delta \beta$ above are the corrections to the Moon's ecliptic longitude and latitude at mean distance $(384,400 \mathrm{~km})$ in order to compensate the 
difference between the center of the reference datum of Watts' lunar limb profile charts and the center of mass of the Moon.

The lunar limb profile irregularity was removed by the corrections given by Watts (1963). In 1994, lunar topographic features were observed by the Clementine lunar mapping mission (Smith et al., 1997), but the Clementine laser altimeter data are too sparse (they only covered narrow meridional strips separated by almost $3^{\circ}$ of selenographic longitude) to replace Watts' data, and the altimeter data for the polar regions were not obtained. Observations not having Watts' limb profile corrections were rejected because these can have systematic effects on the solution.

The lunar positions were calculated from JPL's latest ephemerides DE405 (Standish, 1998). The positions of stars were taken from the Hipparcos Catalogue (ESA, 1997). For the stars not given in the Hipparcos Catalogue the ACT catalog (Urban, 1998) was used. The lunar and stellar positions thus calculated are all claimed to be on ICRS.

The conversion from ICRS to MCEP (Earth's mean equator and mean equinox of J2000) was made using the parameters determined by Chapront $e t$ al., (1999). Precession from J2000 and sidereal time were calculated using the formulas by Williams (1994) that were based on the general precession correction of $-3.266 \mathrm{mas} /$ year to the IAU 1976 value. The nutation was from the ZMOA 1990 solution by Herring (1991). The values of UT1 - UTC and the data for the Earth's polar motion were from BIH and IERS. The geodetic coordinates based on various local geodetic datums were transformed to WGS 84 using the transformation parameters given by NIMA (1997). The geoid heights based on EGM96 were taken into account.

The height $\Delta \sigma$ of the star from the lunar limb, calculated from the observed times were used to solve the correction $\Delta \lambda$ to the lunar ecliptic longitude, the correction $\Delta \alpha$ to the right ascension system of the stars. The rotation $\Delta \epsilon$ of the stars' coordinates around the equinox including their derivatives with respect to time were obtained from the following equation:

$$
\Delta \sigma=\Delta \lambda \cos \beta+\Delta \alpha \cos \lambda \sin \epsilon \cos N+\Delta \epsilon(\cos \lambda \sin \beta \sin N-\sin \lambda \cos N),
$$

where $\lambda$ and $\beta$ are the ecliptic longitude and latitude of the Moon, $\epsilon$ is the obliquity of the ecliptic, and $N$ is the position angle of the event measured from the projection of the ecliptic north pole.

The number of equations is 14,963 and the mean epoch is 1983.9 . The RMS of the residuals is 0 "'201. The solution in mas is as follows:

$$
\begin{aligned}
\Delta \lambda=-94.7 & +0.56(Y-1980), \\
& \pm 2.2 \pm 0.22 \\
\Delta \alpha= & +48.4-4.06(Y-1980), \\
& \pm 12.7 \pm 1.27 \\
\Delta \epsilon= & -17.0+0.67(Y-1980) . \\
& \pm 4.2 \pm 0.43
\end{aligned}
$$

where $Y$ is the year. The values at the epoch 1991.25 of the Hipparcos Catalogue are found to be

$$
\Delta \lambda=-88 \pm 3 \text { mas, }
$$




$$
\begin{aligned}
\Delta \alpha & =+3 \pm 19 \text { mas } \\
\Delta \epsilon & =-9 \pm 6 \text { mas. }
\end{aligned}
$$

The constant term in $\Delta \lambda$ can be regarded as the correction to the value of the difference between the center of the reference datum of Watts' charts and the center of mass of the Moon. Since the value $+00^{\prime \prime} 50$ was adopted for it, the solution suggests that the longitude component of the difference of the centers is +0 ." 41 in the sense that the center in Watts' charts leads the center of mass in the lunar orbit. The constant terms in $\Delta \alpha$ and $\Delta \epsilon$ are found to be not very significant.

The linear terms suggest the following rotation of the Hipparcos reference frame:

$$
\begin{array}{rlrl}
\omega_{x}=(\Delta \epsilon)_{t} & = & +0.67 \pm 0.42 \mathrm{mas} / \text { year } \\
\omega_{y}=(\Delta \lambda)_{t} \sin \epsilon & = & +0.22 \pm 0.09 \mathrm{mas} / \text { year } \\
\omega_{z}=-(\Delta \lambda)_{t} \cos \epsilon+(\Delta \alpha)_{t} & =-4.57 \pm 1.29 \mathrm{mas} / \text { year }
\end{array}
$$

where $(A)_{t}$ means the derivative of $A$ with respect to time. These equations strongly suggest that the differences between equations (1) and (2) are largely due to the remaining rotation of the Hipparcos reference frame.

The quantities $\Delta \alpha$ and $\Delta \delta$ are mainly determined from the ecliptic latitudes of the Moon. Therefore they can be affected by the errors of Watts' charts especially in the regions near the poles of the Moon. The limb profiles of those regions are now being obtained from many lunar grazing occultation observations (Sôma, 1999). By incorporating such profile data, it is expected that the accuracy of the solution would become much higher.

\section{Conclusion}

The lunar occultation analysis suggests much larger errors in the rotation of the Hipparcos reference frame than those given by the Hipparcos team.

Acknowledgments. The author would like to thank the ILOC for providing the occultation observations collected by them, and Mr. Reinhold Büchner in Germany for providing those he obtained from RGO. Thanks are also due to many professional and amateur astronomers who made the observations analyzed in this paper.

\section{References}

Chapront, J., Chapront-Touzé, M., \& Francou, G., 1999, Astron. Astrophys., $343,624$.

European Space Agency, 1997, "The Hipparcos Catalogue," ESA SP.-1200

Feissel, M. \& Mignard, F., 1998, Astron. Astrophys., 331, L33.

Fricke, W. et al., 1988, Veröff. Astron. Rechen-Inst. Heidelberg, No. 32.

Herring, T.A., 1991, in Reference Systems, Proceedings of the 127th Colloquium of the IAU (eds. Hughes, J.A., Smith, C.A., \& Kaplan, G.H.), USNO, Washington D.C., 157. 
McCarthy, D.D. \& Luzum, B.J., 1991, Astron. J., 102, 1889.

Miyamoto, M. \& Sôma, M., 1993, Astron. J., 105, 691.

National Imagery and Mapping Agency, 1997, NIMA TR8350.2.

Smith, D.E., Zuber, M.T., Neumann, G.A., \& Lemoine, F.G., 1997, J. Geophys. Res., 102, 1591.

Sôma, M., 1985, Celest. Mech., 35, 45.

Sôma, M., 1999, Publ. Natl. Astron. Obs. Japan, 5, 99.

Standish, E.M., 1998, JPL IOM 312.F-98-048.

Urban, S.E., Corbin, T.E., \& Wycoff, G.L., 1998, Astron. J., 115, 2161.

Watts, C.B., 1963, Astron. Papers Am. Ephemeris, 17, U.S. Government Printing Office, Washington, D.C.

Williams, J.G., Newhall, X X, \& Dickey, J.O., 1991, Astron. Astrophys., 241, L9.

Williams, J.G., 1994, Astron. J., 108, 711. 